\title{
The Optimum Programming Model of Toll Plaza Based on Fluid Mechanics
}

\author{
Feng Zhang \\ North China Electric Power University, Baoding 071000, China \\ 445324940@qq.com
}

Keywords: Toll Plaza; Fluid Mechanics; Optimum Programming Model; VISSIM

Abstract.This thesis builds the optimum programming model based on fluid mechanics. Under the premise of a fixed number of lanes, L, with the shortest passing time as the optimization target, this thesis determines the best plan for the number of tollbooths, $n$, which provides the preliminary basis for the design of the new toll station. And verify the accuracy of the results with the help of VISSIM.

\section{Introduction}

The toll station, served as the bond to highways, has always been the limit to the capacity of the highway. With the traffic increasing, the traditional mode of type toll station has been unable to meet the needs of network construction. Therefore, how to reasonably analyze and simulate the toll stations has become an important factor to improve the highway transport capacity.

\section{The Fluid Mechanics Model}

In order to get the performance analysis of traditional toll plaza, first I study the relationship between $\mathrm{n}$ and $\mathrm{L}$. That is, how to determine $\mathrm{n}$ on the basis of a known $\mathrm{L}$. In order to solve this problem, the article believes that the value of $n$ should be determined when the lanes are in heavy traffic, so that I can shorten the length of queues in a traffic jam. In the case of a large traffic flow, the expressway has the following features:

a. It is a fully closed system with full interchange and strict access control;

b. It is a car-specific system with speed limit;

c. It has central reservations and cars run on both sides of the central reservation.

Hence, it is possible to use the theory of fluid mechanics to simulate the traffic flow as a continuous fluid flow, that is, establish the model of the fluid mechanics to study the features of the traffic flow at the toll station.

The process of a vehicle passing through a toll station can be divided into three sections: the entering section, the waiting for service section and the exiting section, as shown in Figure 1:

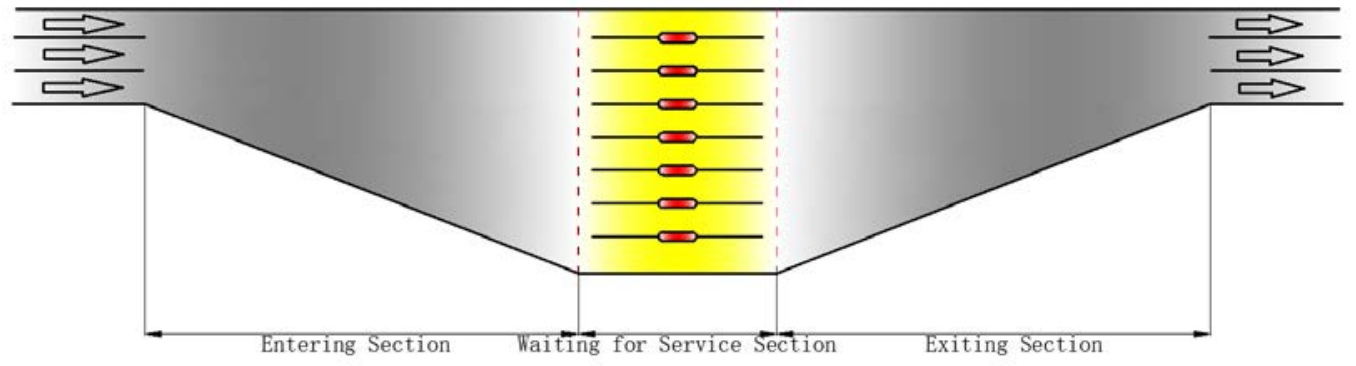

Fig.1 The process of a vehicle passing through a toll station

Correspondingly, the total model can be divided into three sub-models. Each sub-model is connected to each other by a traffic flow q and a flow velocity v. Each section corresponds to a period of time, hence the total time is described as the following equation ${ }^{[1]}$ :

$$
T=T_{n}+T_{w}+T_{e}
$$

Where:

$T_{n}$ describes the average time traffic flow spends in the entering section; 
$T_{w}$ describes the average time traffic flow spends in the waiting for service section;

$T_{e}$ describes the average time traffic flow spends in the exiting section;

$T$ describes the total average time traffic flow spends in the toll plaza.

\section{Modeling of the entering section.}

I use the theory of fluid mechanics to model the traffic flow as a continuous flow. I assume that the traffic flow is a free flow and it enters the toll plaza in an initial traffic flow of $q_{0}$ and at an initial speed of $v_{0}$.

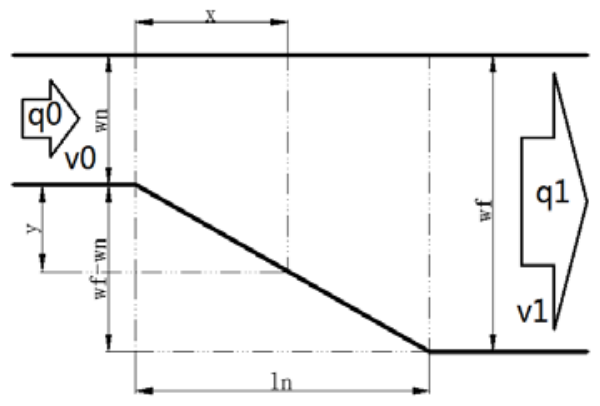

Fig. 2 The figure of the entering section

By using fluid mechanics and geometric knowledge, we can get:

$$
T_{n}=\frac{n w_{n} a_{1}}{v_{0}\left(n d-w_{n}\right)} \ln \left(\frac{n d}{w_{n}}\right)
$$

Where,

$n$ describes the number of tollbooths in a toll plaza;

$w_{n}$ describes the width of the entrance of the entering section;

$a_{1}$ describes a coefficient whose value is usually $0.02^{[2]}$;

$v_{0}$ describes the initial speed of traffic flow;

$d$ describes the width of each tollbooth in a toll plaza.

\section{Modeling of the waiting for service section.}

According to the fluid mechanics model, the traffic flow is evenly distributed to each toll intersection, hence the traffic flow in each toll intersection is $q_{1} / n$. On the basis of queuing theory, each toll intersection obeys the $\mathrm{M} / \mathrm{M} / 1$ system, hence the waiting for service section is a number of $\mathrm{M} / \mathrm{M} / 1$ systems. The waiting time can be calculated according to the following formula:

$$
T_{\mathrm{w}}=\frac{n}{n \theta-q_{1}}
$$

Where,

$q_{1}$ describes the traffic flow at the export of the entering section;

$\theta$ describes the average service rate of a tollbooth.

\section{Modeling of the exiting section.}

This section is similar to the entering section and we can get:

$$
T_{e}=\frac{n^{2} d a_{1}}{v_{2}\left(n d-w_{u}\right)} \ln \frac{w_{u}}{n d}
$$

Where,

$v_{2}$ describes the speed of traffic flow at the entrance of the exiting section;

$w_{u}$ describes the width of the export of the exiting section.

The solution process for the model.

Based on the derivation above, the total time of a vehicle passing through the toll plaza is the objective function, and its formula is as follows:

$$
T=T_{n}+T_{w}+T_{e}=\frac{n w_{n} a_{1}}{v_{0}\left(n d-w_{n}\right)} \ln \frac{n d}{w_{n}}+\frac{n}{n \theta-q_{1}}+\frac{n^{2} d a_{1}}{v_{2}\left(n d-w_{u}\right)} \ln \frac{w_{u}}{n d}
$$

The values of the parameters are shown in the following table: 
Table 1 The values of parameters

\begin{tabular}{ccccccc}
\hline$w_{n}[\mathrm{~km}]$ & $w_{u}[\mathrm{~km}]$ & $d[\mathrm{~km}]$ & $E(\mu)[\mathrm{pcu} / \mathrm{h}]$ & $E\left(q_{0}\right)[\mathrm{pcu} / \mathrm{h}]$ & $v_{0}[\mathrm{~km} / \mathrm{h}]$ & $v_{2}[\mathrm{~km} / \mathrm{h}]$ \\
\hline 0.015 & 0.015 & 0.005 & 600 & 3400 & 40 & 20 \\
\hline
\end{tabular}

$E(\mu)$ and $E\left(q_{0}\right)$ are respectively the mathematical expectations of $\theta$ and $q_{0}$.

When the value of $n$ varies, the diagram between $\mathrm{T}[h]$ and $\mathrm{n}$ is shown below:

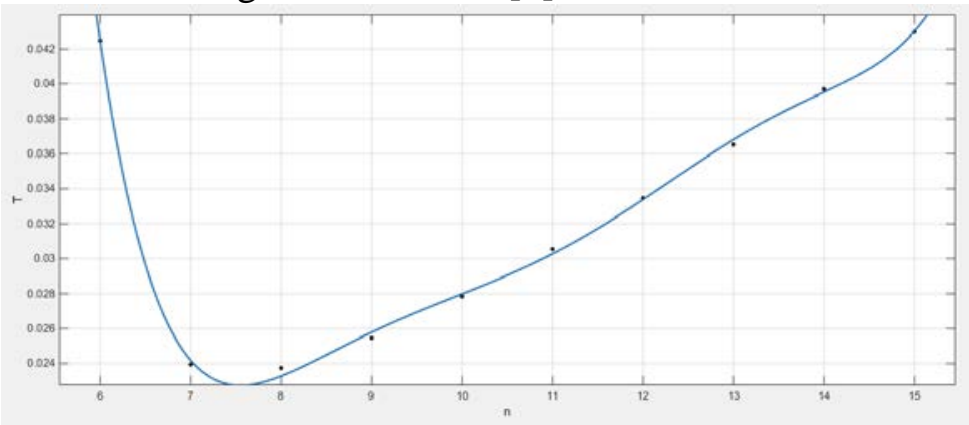

Fig.3 The n-T diagram

Therefore, in the case of a three-lane highway, when the value of $n$ is 8 , the average waiting time of a vehicle is the shortest. That is, the average number of vehicles in the queuing system is 24 , and the average time of queuing system is $21 \mathrm{~s}$.

\section{Simulations on VISSIM}

In order to verify the calculation results, I simulate the total average time traffic flow spends in the toll plaza (T) on VISSIM.

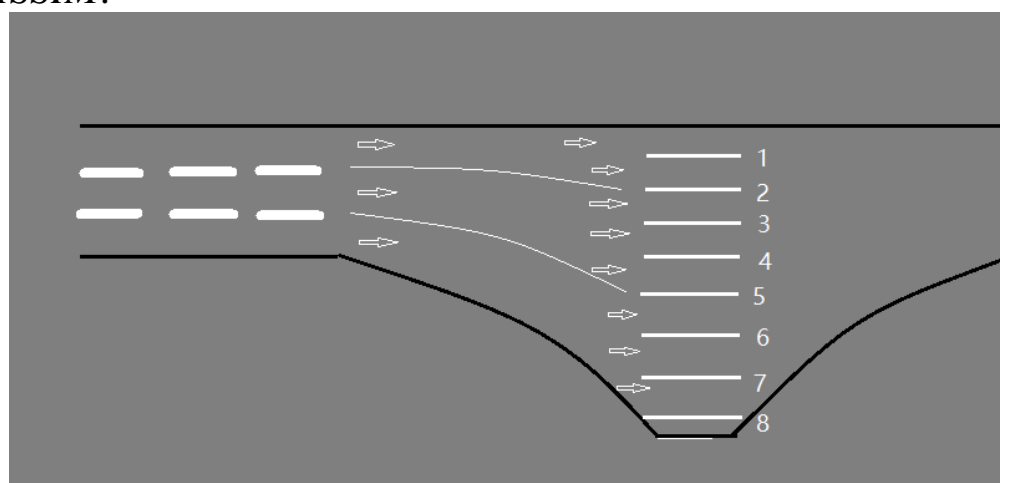

Fig. 4 The sketch of entering section

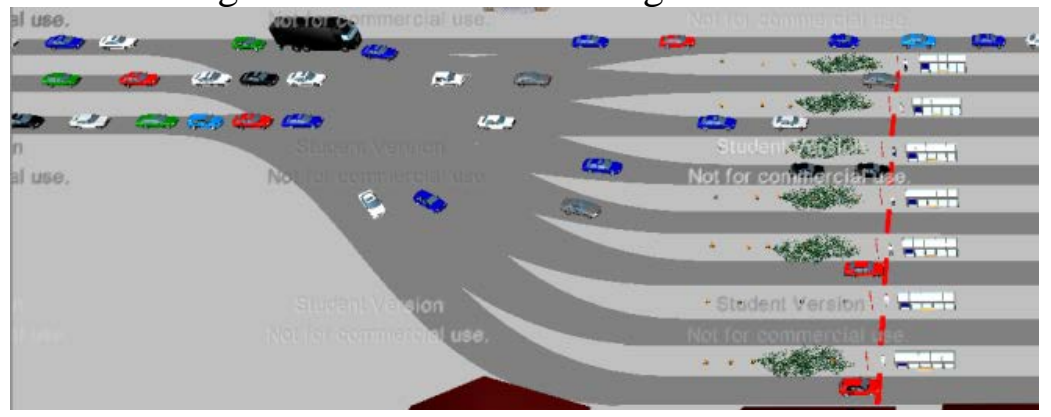

Fig. 5 The simulation

In order to design the signal controller, assume that the average service time is $18 \mathrm{~s}$, and set the release time to $2 s$. So the proportion of time for red traffic lights to that for green traffic lights is 18:2 with a control cycle of 20s. Meanwhile, I ensure the randomness of different signal controllers. The signal controllers I set are as follows: 


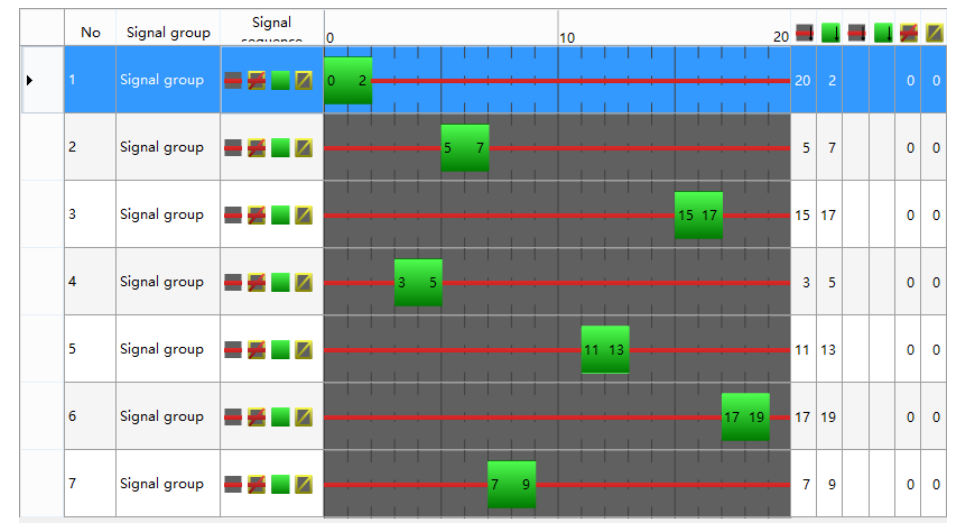

Fig. 6 The signal controllers

Based on the variables of the models above, I set the network parameters and add the queue counter elements to obtain the time $T$.

Table 2 The simulation results

\begin{tabular}{ccccccccc}
\hline Number of tollbooths & 6 & 7 & 8 & 9 & 10 & 11 & 12 & 13 \\
\hline Average time[s] & 140 & 72 & 25 & 90 & 103 & 108 & 122 & 130 \\
\hline
\end{tabular}

By comparing the simulation results with Figure 3, we can find that there is little difference between them, and the accuracy of the model can be verified.

\section{Conclusions}

This thesis builds the optimum programming model based on fluid mechanics. By modeling, this article calculates the passing time of transport flow. Then with a known $\mathrm{L}$, set the shortest passing time as the optimization target and get the best plan for the number of tollbooths, $\mathrm{n}$. In the end, simulate the toll station. By comparing the passing time between the simulation and modeling results, I verify the accuracy of the model.

\section{References}

[1] Chunlei Wu, Yulin Chang, Configuration Research of Toll-gate on Freeway [J]. Highway Engineering, 2008 (5):172-175

[2] Yimei Luo, Yi Song, Baoquan Ji. Study on Improvement of Passthrough Capacity of Mainline Toll-stations on Expressways [J]. Logistics Technology, 2013(5):285-330 